\title{
Impacto de una vía clínica para el pie diabético en un hospital general
}

\author{
D. A. MARTÍNEZ, J. L. AGUAYO, G. MORALES, L. M. AGUIRÁN, F. ILLÁN \\ Servicio de Cirugía General y Aparato Digestivo. Hospital General Universitario \\ Morales Meseguer. Murcia
}

\begin{abstract}
IMPACT OF A CLINICAL PATHWAY FOR THE DIABETIC FOOT IN
\end{abstract} A GENERAL HOSPITAL

\section{RESUMEN}

Objetivo: Las úlceras, infecciones y la isquemia del pie son las principales causas de hospitalización del paciente diabético y con mucha frecuencia el motivo de la amputación del miembro inferior. El objetivo de esta investigación consiste en evaluar los resultados de una vía clínica multidisciplinaria diseñada por el conjunto de especialistas de distintos departamentos de nuestro hospital, así como del personal de enfermería asociado, implicados en el tratamiento de esta patología.

Pacientes y métodos: Hemos analizado la evolución de una serie de indicadores clínicos y socioeconómicos comparando, en los pacientes ingresados con el diagnóstico de pie diabético complicado, el periodo previo a la implantación de la vía clínica (años 1998, 1999 y 2000) con el periodo posterior a dicha implantación (años 2001 y 2002). Se evalúa en cada grupo: el porcentaje de amputaciones mayores y menores realizadas, la mortalidad intrahospitalaria, la estancia media, el porcentaje de reingresos en 30 días, el porcentaje de arteriografías realizadas, el porcentaje de pacientes controlados por rehabilitación, y el coste económico de los procesos individuales, así como el costo global. En el grupo incluido en la vía clínica se llevó a cabo además una encuesta de satisfacción. La comparación estadística se realizó mediante la Chi cuadrado.

Resultados: No encontramos diferencias ni en la estancia media, ni en la mortalidad intrahospitalaria. Si encontramos un claro aumento en el porcentaje de arteriografías (del 3,5\% se paso al 13\%) y en el porcentaje de pacientes controlados por rehabilitación (del 19,8\% se paso al $84,3 \%$ ). La tasa de amputaciones mayores de los pacientes no incluidos en la vía clínica paso de $17,4 \%$ a $9,7 \%$ tras la implantación de dicha vía. La tasa de reingresos a 30 días disminuyó de 9,3 a 6,5\%. El coste económico global y el coste medio por paciente ha sido inferior tras la implantación de la vía clínica. La encuesta de satisfacción de este último grupo ofreció una satisfacción global del $95 \%$.

Conclusiones: Los resultados obtenidos sugieren que la aproximación al diagnóstico y tratamiento al pie diabético complicado mediante esta vía clínica multidisciplinaria mejora la evolución del paciente hospitalizado, disminuyendo el número de amputaciones mayores, sin prolongarse la estancia media y manteniendo, o incluso disminuyendo, el coste económico global.

PALABRAS CLAVE: Pie diabético. Vías clínicas. Amputación de extremidad. Calidad asistencial.

\section{ABSTRACT}

Objective: The ulcers, infections and isquemia of the foot are the main causes of hospitalisation of the diabetic patient and, very frequently, they are reason for the amputation of the limb. The objective of this investigation consists of evaluating the results of a multidisciplinary clinical pathway designed by the set of specialists of different departments from our hospital, as well as of the associated personnel of infirmary, implied in the management of this pathology.

Patients and methods: We have analysed the evolution of a series of clinical and socio-economic indicators comparing, in the patients admitted with diagnose of complicated diabetic foot, the previous period to the implantation of the clinical pathway years 1998, 1999 and 2000) with the later period to this implantation (years 2001 and 2002). It is evaluated in each group: the percentage of greater and smaller amputations, mortality, the average stay, the percentage of readmitances in 30 days, the percentage of arteriographies, the percentage of patients controlled by rehabilitation, and the economic cost of the individual processes, as well as the global cost. In the group including in the clinical pathway it was carried out, in addition, a satisfaction survey. The statistical comparison was made by means of the Chi square test.

Results: We did not find differences as far as the average stay, nor to intrahospitable mortality. If we found a clear increase in the percentage of arteriographies (of the $3.5 \%$ passage to $13 \%$ ), and in the percentage of patients controlled by rehabilitation (of the $19.8 \%$ passage to the $84.3 \%$ ). The rate of greater amputations of the patients not including in the clinical pathway change from $17.4 \%$ to $9.7 \%$ after the implantation of this route. The rate of readmitances to 30 days diminished from 9.3 to $6,5 \%$. The global economic cost and the average cost by patient have been inferior after the implantation of the clinical route. The survey of satisfaction of this last group offered a global satisfaction of $95 \%$.

Conclusions: The results suggest that the approach to the diagnose and treatment on the complicated diabetic foot by means of this multidisciplinary clinical pathway improves the evolution of the hospitalised patient, diminishing the number of greater amputations, without extending the average stay and maintaining, or even diminishing, the global economic cost.

KEY WORDS: Diabetic foot. Clinical pathway. Amputation of extremity. Welfare quality.

Martínez, DA, Aguayo JL, Morales G, Aguirán LM, Illán F. Impacto de una vía clínica para el pie diabético en un hospital general. An Med Interna (Madrid) 2004; 21: 420-424. 


\section{INTRODUCCIÓN}

La incidencia de patología severa en los pies de los pacientes diabéticos viene a ser del 5-10\% en esta población, incrementándose con la edad y el desarrollo evolutivo de la diabetes. El pie diabético es la principal causa de hospitalización de estos pacientes, ya sea por la aparición de ulceración, isquemia o infección. Así, en los países occidentales, cada año hasta el $10 \%$ de los pacientes que sufren problemas establecidos en los pies requieren un largo ingreso hospitalario y en muchas ocasiones, algún grado de amputación (1-4). Muchas de estas úlceras pueden curar sin necesidad de amputación o cuando ésta es necesaria, puede ser más conservadora de la extremidad si se realiza un tratamiento adecuado de la infección, se evita cargar peso sobre la zona afectada y se restablece la irrigación arterial en el pie isquémico(5).

El manejo hospitalario de estas lesiones del pie diabético, cuando se complican, genera un alto costo económico y social. Por otra parte, el equipo sanitario implicado en la atención de estos enfermos es pluridisciplinario (unidad de urgencias, cirujanos generales y/o vasculares, endocrinólogos, rehabilitadores, radiólogos, enfermeros, fisioterapéutas, podólogos, etc.), circunstancia que puede condicionar un alto grado de variabilidad clínica en su manejo.

Las vías clínicas son planes asistenciales para un proceso clínico de curso más o menos predecible. Integran varios protocolos de actuación en las sucesivas fases del proceso, para diferentes profesionales y en las distintas unidades asistenciales implicadas. Se basan en datos de la medicina basada en la evidencia y requieren un amplio consenso. Su objetivo es mejorar el trabajo multidisciplinario en equipo, intentando optimizar los resultados cínicos y el consumo de recursos (69). El objetivo de este trabajo es analizar el impacto que en los resultados, costes y satisfacción del paciente, ha supuesto la puesta en marcha de una vía clínica para el pie diabético en un servicio de cirugía general.

\section{PACIENTES Y MÉTODOS}

Los criterios de ingreso hospitalario para el pie diabético fueron establecidos previamente: a) úlcera que sobrepasa el celular subcutáneo, con o sin isquemia asociada; b) infección con celulitis de extensión superior a los $2 \mathrm{~cm}$; y c) gangrena isquémica que afecta a los dedos o al resto del pie.

Los datos se obtuvieron retrospectivamente del sistema de información del Hospital Morales Meseguer, incluyendo todos los pacientes ingresados en el Servicio de Cirugía General y Aparato Digestivo, desde enero de 1998 a diciembre de 2002, usando los códigos diagnósticos de la $9^{a}$ revisión, modificación clínica, de la clasificación internacional de enfermedades (CIE-9-MC): 250,xx (diabetes mellitus) y sus complicaciones, 707,1 (úlcera de miembros inferiores salvo decúbito), 785,4 (gangrena), 250,7 (diabetes con trastornos circulatorios periféricos), 277-278 (celulitis con o sin complicaciones). Se excluyeron los casos en los que éstos diagnósticos no fueron el principal, constando sólo como secundarios.

Se han comparado dos grupos: El grupo 1, antes de la implantación de la vía clínica (años 1998-2000), estuvo formado por 227 enfermos de los cuales 137 eran varones (60\%) y 90 mujeres (40\%), con una edad media de 67 años (28-92). El grupo 2, posterior a la implantación de la vía (años 2001-
2002), estuvo compuesto por 166 pacientes de los cuales 96 eran varones $(58 \%)$ y 70 mujeres $(42 \%)$, con una edad media de 68 años (30-89). La estructura y contenido de la vía clínica del pie diabético de nuestro hospital ha sido publicada previamente (10).

Estudiamos en cada grupo: a) Resultados clínicos: número de amputaciones mayores (supra e infracondíleas) y menores (digitales y transmetatarsianas), mortalidad intrahospitalaria, y reingresos en 30 días; b) Criterios de calidad: estancia media, número de casos con estudio arteriográfico con o sin angioplastia y número de pacientes con tratamiento por el servicio de rehabilitación; c) Costo tanto por proceso como anual (sin ajuste inflacionario). El sistema de contabilidad analítica de nuestro hospital obtiene el "coste por paciente" a partir de la suma de los costes de los productos intermedios realizados a cada paciente y el "coste por proceso" como la suma de los costes de los pacientes individuales incluidos en el mismo; d) Encuesta de satisfacción, efectuada únicamente en el grupo 2.

La comparación estadística se realizó mediante el test de la chi-cuadrado.

\section{RESULTADOS}

\section{CLÍNICOS}

En el primer periodo, 56 pacientes $(24 \%)$ sufrieron una amputación mayor y 82 (36\%) una amputación menor, mientras que en el segundo periodo 26 pacientes $(15,6 \%)$ requirieron una amputación mayor y $69(44,23 \%)$ una resección menor (Tabla I). Al descontar las amputaciones realizadas en las primeras horas tras el ingreso, de realización obligada por presentar gangrena avanzada establecida (20 casos en el grupo 1 y 12 casos en el grupo 2), los resultados son los que se ofrecen en la Figura 1, con frecuencias de 17,4\% antes de la vía clínica y $9,7 \%$ tras la misma $(\mathrm{p}<0,05)$.

La mortalidad ha permanecido estable, siendo del $5 \%$ en el primer periodo, y del 4,2\% en el segundo periodo.

La tasa de reingresos antes de 30 días fue del 9,3\% (21 casos) en el primer periodo y del $6,6 \%$ (11 casos) en el segundo.

\section{CRITERIOS DE CALIDAD}

La estancia media se ha mantenido fija entre el 11-11,7 días, con la evolución que se muestra en la Figura 2.

La realización de estudios arteriográficos se expone en la Tabla II. Se aprecia cómo se ha multiplicado la práctica de los mismos, desde el 3,5\% del primer periodo al $12,6 \%$ del segundo.

TABLA I NÚMERO DE AMPUTACIONES TOTALES

\begin{tabular}{ccc}
\hline Años & $1998-2000$ & $2001-2002$ \\
\hline Amputación mayor & $56(24 \%)$ & $26(16,6 \%)$ \\
Amputación memor & $82(36 \%)$ & $69(44,2 \%)$ \\
Sin Amputación & $89(40 \%)$ & $71(59,2 \%)$ \\
Pacientes & 227 & 166 \\
\hline
\end{tabular}




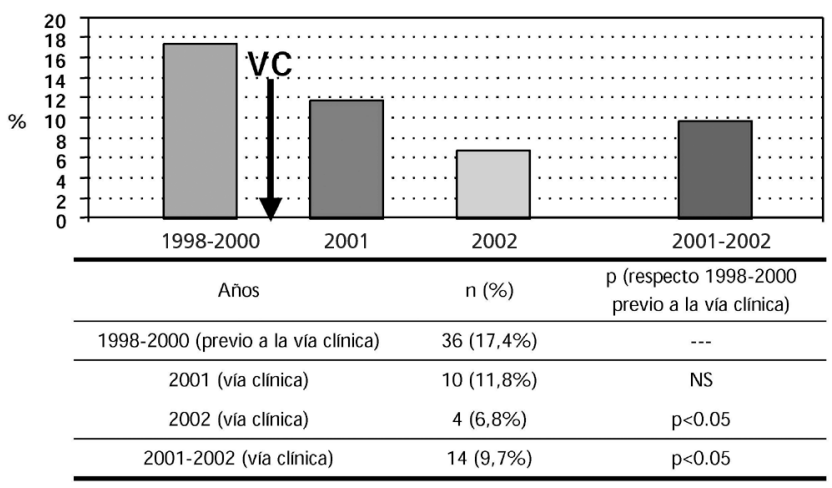

Fig. 1. Número de amputaciones mayores excluidas las realizadas en urgencias al ingreso.

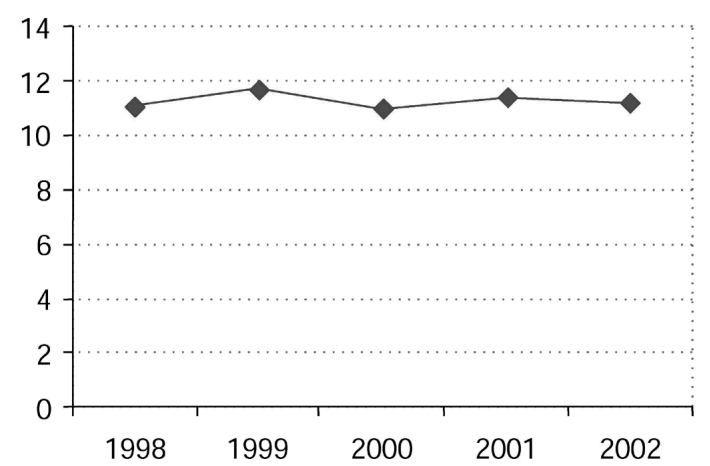

Fig. 2. Estancia media anual (días).

\begin{tabular}{ccc} 
TABLA II & \\
\hline NÚMERO DE ESTUDIOS ARTERIOGRÁFICOS \\
\hline Años & $\begin{array}{r}\text { p (respecto 1998-2000 } \\
\text { previo a la vía clínica) }\end{array}$ \\
\hline 1998-200 (previo a vía clínica) & $8(3,5 \%)$ & $\cdots$ \\
$2001-2002$ (vía clínica) & $21(13 \%)$ & p<0,01 \\
\hline
\end{tabular}

El número de pacientes que fueron controlados por el servicio de rehabilitación ha pasado de tasas inferiores al $20 \%$ a porcentajes del 84\% (Tabla III).

\begin{tabular}{|c|c|c|}
\hline \multicolumn{3}{|c|}{ TABLA III } \\
\hline \multicolumn{3}{|c|}{$\begin{array}{c}\text { NÚMERO DE PACIENTES CONTROLADOS } \\
\text { POR REHABILITACIÓN }\end{array}$} \\
\hline Años & & $\begin{array}{l}\text { p (respecto 1998-2000 } \\
\text { previo a la vía clínica) }\end{array}$ \\
\hline $\begin{array}{c}\text { 1998-200 } \\
\text { (previo a vía clínica) }\end{array}$ & $45(19,8 \%)$ & --- \\
\hline $\begin{array}{l}2001-2002 \\
\text { (vía clínica) }\end{array}$ & $140(84,3 \%)$ & $p<0,01$ \\
\hline
\end{tabular}

COSTES

Los costes medios de los GRD analizados, así como el coste global de cada uno, el de la totalidad anual y el coste medio del paciente se muestran en la Tabla IV en los dos periodos de tiempo, antes y después de la implantación de la vía clínica. Como vemos, el coste medio por paciente, aún sin realizar ajuste inflacionario alguno, ha sido inferior tras la implantación de la vía clínica. La evolución anual de los costes se muestra en la Figura 3.

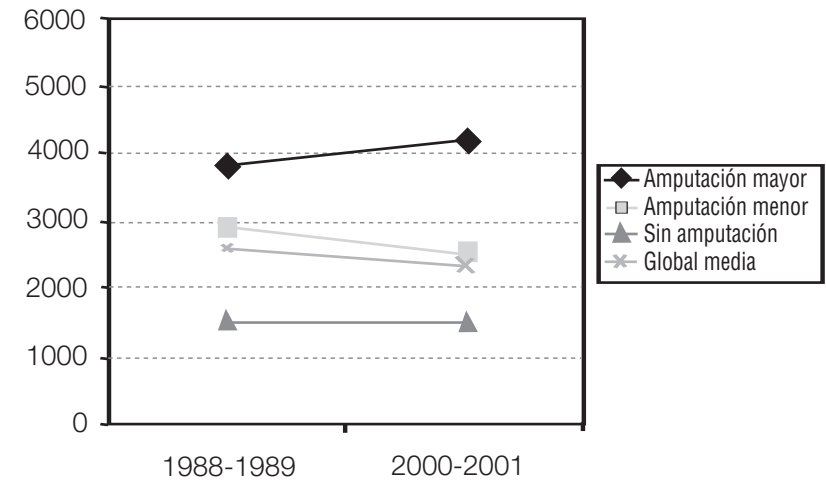

Fig. 3. Coste GRD (€).

TABLA IV

COMPARACIÓN DE COSTES ANTES Y DESPUÉS DE LA PUESTA EN MARCHA DE LA VC

\begin{tabular}{lccccc}
\hline Periodo & Costo medio & $N^{0}$ pacientes & $\begin{array}{c}\text { Costo global } \\
\text { del GRD (€) }\end{array}$ & $\begin{array}{c}\text { Coste global del } \\
\text { conjunto de GRD (€) }\end{array}$ & $\begin{array}{c}\text { Coste medio por } \\
\text { paciente }(€)\end{array}$ \\
\hline \multirow{2}{*}{$1998-2000$} & Sin amputación (1) & 1.500 & 89 & 133.500 & 584.100 \\
& Amputación menor (2) & 2.900 & 82 & 237.800 & 2.573 \\
& Amputación mayor (3) & 3.800 & 56 & 12.800 & 388.200 \\
\hline
\end{tabular}




\section{ENCUESTA DE SATISFACCIÓN}

El porcentaje de cumplimentación fue del $60 \%$ y, el de satisfacción global, del $95 \%$. Otros detalles se muestran en la Tabla V.

\section{TABLA V}

\section{ENCUESTA DE SATISFACCIÓN}

\begin{tabular}{lc}
\hline Cumplimentación & $60 \%$ \\
Satisfacción global & $95 \%$ \\
Control dolor leve & $72 \%$ \\
Control dolor moderado & $18 \%$ \\
Control dolor intenso & $9 \%$ \\
Quejas (todas referidas al apartado de hostelería) & $36 \%$ \\
Otras respuestas negativas (desconocimiento & $27 \%$ \\
del nombre del médico o enfermera) &
\end{tabular}

\section{DISCUSIÓN}

Las complicaciones del pie en los diabéticos suponen una parte importante de la actividad clínica de un servicio de cirugía (habitualmente cirugía general) en un hospital de área. Suele afectar a pacientes diabéticos de edad avanzada, muestra una elevada mortalidad, concluyen con grados variables de amputación en un buen número de casos y requieren un importante consumo de recursos. Se trata de un problema frecuente que se caracteriza por implicar a un gran número de diferentes profesionales de salud, tanto de especialidades médicas (multidisciplinar), como de enfermería (interdisciplinar). Este último aspecto, aunque positivo en general, puede favorecer un alto grado de variabilidad asistencial, así como una tendencia a diluir las responsabilidades en cuanto a los resultados del tratamiento: muchos profesionales lo tratan sin que ninguno se considere el responsable principal. Por otra parte, aunque la diabetes afecta sólo al 5\% de la población (1, 2), más de la mitad de las amputaciones del miembro inferior se realizan a causa del pie diabético $(3,4)$, estando precedidas en $85 \%$ de los casos por una úlcera en el pie (3) que permanece bajo tratamiento varios meses o incluso años. Durante el lapso de tiempo entre la aparición de la úlcera en el pie y su cicatrización, con o sin algún tipo de amputación, se suceden distintos tipos de tratamientos con mayor o menor éxito. Con el fin de disminuir la variabilidad clínica en cuanto al manejo diagnóstico y terapéutico intrahospitalario por los distintos profesionales implicados, nos planteamos la realización de una vía clínica para el pie diabético como un método de consenso que implicara a todo el grupo de trabajo (10).

El objetivo más importante de esta vía clínica es conseguir una reducción en la tasa de amputaciones mayores en el paciente diabético. La incidencia de amputaciones no traumáticas en España se ha estimado casi 30 veces mayor en pacientes diabéticos que en los que no lo son (4). La reducción de esta elevadísima tasa de amputación mayor sólo será un hecho cuando se realicen los adecuados programas preventivos a nivel primario, secundario y terciario. La prevención primaria trata de impedir la aparición de la neuropatía periférica diabética, mediante el mantenimiento de unos niveles adecuados de glucemia, así como la aparición de la enfermedad arterial periférica mediante el control de los factores de riesgo de la arterioesclerosis. Cuando ya han aparecido la neuropatía y/ó la angiopatía, encontrándonos por tanto frente a un "pie de riesgo", la prevención secundaria mediante el uso de un calzado adecuado y el mejor tratamiento médico disponible para la enfermedad vascular periférica, intentará evitar la aparición de la úlcera diabética. Estos dos aspectos de la prevención se realizan en atención primaria, es decir en el medio extrahospitalario. La prevención terciaria tiene como objetivo evitar la amputación en pacientes con úlcera abierta, haciéndose necesario en este marco clínico el concurso especializado. Cuando nos encontramos con estadios aún más avanzados de la enfermedad, con infección sobreañadida y/o isquemia crítica la "amenaza de pérdida de la extremidad inferior" es ya una realidad inminente y el paciente suele precisar el ingreso hospitalario. Esta situación clínica cumple casi todos los criterios que se han recomendado para la aplicación de las vías clínicas intrahospitalarias.

La tasa de amputación mayor en pacientes diabéticos que precisan hospitalización por un problema relacionado con infección y/ó isquemia del pie, cuando se incluyen todos los pacientes, alcanza cifras superiores al 20\% $(11,12)$, aunque la edad media de los pacientes que precisan amputación mayor es algo superior a los 75 años, es decir se concentran en el paciente con pie diabético y avanzada edad. Algunas de estas amputaciones se realizan tras las primeras horas después del ingreso por acudir a urgencias con un cuadro de gangrena isquémica avanzada de la extremidad (tabla III). Estos casos no siguen obviamente la vía clínica, por lo que deben excluirse de la comparación entre los dos periodos de tiempo. La tasa de amputación mayor se redujo desde el 17,4\% antes de aplicar la vía clínica, al 9,7\% tras la aplicación de dicha vía.

Los reingresos, con posibilidad de haberse vistos aumentados por la reducción en el número de amputaciones, no han crecido e incluso existe una ligera disminución. Este detalle es importante, porque refleja que no ha habido una insuficiencia de tratamiento.

La estancia media, sobre la que podíamos temer un alargamiento debido al esfuerzo en el salvamento de la extremidad y el mayor número de exploraciones complementarias requerido, se ha mantenido estable, sobre los 11 días. Pueden compararse estas cifras, en España, con los datos aportados por Monereo (13) para el que la estancia media de la angiopatía periférica en un hospital del grupo 3 del Insalud, fue de 19'8 días y representó la más prolongada de todas las relacionadas con complicaciones de la diabetes mellitus. En USA (14), la estancia media calculada varía según el GRD y la compañía aseguradora, oscilando entre 6,8 y 19,9 días para los siguientes grupos: 263-injerto cutáneo o desbridamiento para celulitis o úlcera con complicaciones-; 264 -igual que 263 pero sin complicaciones-; 271-úlceras cutáneas-; y 287-injerto cutáneo y desbridamiento de herida para desórdenes endocrinos, metabólicos y nutricionales-.

El número de pacientes que recibieron examen arteriográfico y que fueron estudiados por rehabilitación crecieron rotundamente tras la instauración de la vía clínica, una muestra más de la mejora en el manejo del pié diabético.

Cuando se intentan cuantificar los costes que representa el pie diabético, la mayoría de los estudios se refieren sólo a los costes directos (visitas, hospitalizacion y procedimientos, medicación, etc.), mientras que los indirectos (pérdida de 
ingresos o pagos por baja laboral, cargas familiares, dolor o sufrimiento) son mucho más difíciles de medir. Referido a los costes directos, Ramsey (15) ha cifrado el gasto medio del cuidado de una úlcera del pie diabético, en unos 4,5 millones de pesetas (27.000). Apelqvist (16), calculó el costo del tratamiento de la úlcera del pie diabético cuando se conseguía la curación sin amputación (6.600 \$ USA) frente al costo de la curación con amputación (44.700 \$ USA), incluyendo costos tanto intra como extrahospitalarios. En este estudio, en el primer caso, el $40 \%$ de los costos fueron en el marco intrahospitalario, mientras que, cuando se precisó algún tipo de amputación, los costes intrahospitalarios alcanzaron el $80 \%$ del total. Otra muestra de la enorme repercusión económica es el hecho de que en USA, los seguros privados reembolsaron a los hospitales por el GRD 271 (úlcera dérmica) entre 4.862 y 8.988 \$ por caso, considerando una estancia media entre 8,8 y 11,4 días (15).

En España, se ha estudiado el coste directo de la úlcera del pie diabético en un Area de Salud (17), siendo la mayor proporción del gasto $(70 \%)$ atribuible a la hospitalización. La antibioterapia supuso una parte importante del costo del tratamiento, tanto intra como extrahospitalario.

Temíamos un incremento notable de los costes al instaurar un mayor nivel de cuidados y de esfuerzo en el salvamento de la extremidad. Sin embargo, los costes hospitalarios por pro- ceso se mantuvieron estables o, particularmente en el caso de la amputación mayor, disminuyeron globalmente.

Los resultados de la actividad clínica deben incluir valoración de la satisfacción del paciente o familiares. No contamos con referencias previas a la instauración de la vía, pero el hecho de encontrar niveles de satisfacción global positiva del 95\% nos lleva a defender que el mayor grado de coordinación y mejores resultados propician la percepción de la calidad, incluso en un estado patológico con tanto sufrimiento asociado como el pie diabético.

La única publicación que hemos encontrado en la revisión de la literatura consultada respecto a la utilización de la vía clínica intrahospitalaria en el pie diabético, ha sido la de Crane y Werber (18). El grupo de pacientes que siguió la vía clínica se acompañó de una tasa inferior de amputaciones mayores puesto que disminuyeron desde cifras en torno al $23 \%$ o $29 \%$ (sin vía clínica), hasta cifras en torno al $7 \%$ cuando se siguió dicha vía.

En conclusión, la utilización de una vía clínica para el pie diabético ha conseguido una mejora del proceso: se ha reducido el porcentaje de amputación mayor, se ha incrementado el control arteriográfico y rehabilitador, sin elevar la estancia media ni el índice de reingresos. Los costes se han mantenido estables o incluso han disminuido. La satisfacción de pacientes y familiares ha sido buena.

\section{Bibliografía}

1. Consenso para la atención a las personas con diabetes mellitus en España. Revisión crítica y nuevas perspectivas. Madrid, Centro de Publicaciones del Ministerio de Sanidad y Consumo, 1995.

2. Reiber GE, Boyko E, Smith DG. Lower extremity ulcers and amputations in individuals with diabetes. In: Harris MI (ed) Diabetes in America. 2nd Ed. Bethesda, MD: National Institutes of Health 1995: 409-427. National Institutes of Health Publication No 95-1468.

3. Reiber GE. The epidemiology of diabetic foot problems. Diabetic Medicine 1998; 13: S6-S11.

4. Calle-Pascual AL, Redondo MJ, Ballesteros M, Martínez-Salinas MA, Díaz JA, De Matías P. Nontraumatic lower extremity amputations in diabetic and non diabetic subjects in Madrid, Spain. Diabetes \& Metabolism (Paris) 1997; 23: 519-23.

5. American Diabetes Asociation: Consensus Development Conference on diabetic foot wound care. Diabetes Care 1999; 22: 1354-1360.

6. Carrasco G, Ferrer J. Las vías clínicas basadas en la evidencia como estrategia para la mejora de la calidad: metodología, ventajas y limitaciones. Rev Calidad Asistencial 2001; 16: 199-207.

7. Campbell H, Hotchkiss R, Bradshaw N. Integrated care pathways. GMJ 1998; 316: 133-7.

8. García J, Díez J, Chamorro L, Navas A, Franco A, Arribas J. Vías clínicas. Medicina Preventiva 1999; 1: 28-39.

9. Viana A, Fernández J, Sainz A. Descripción y evaluación de las vías clínicas en una red de hospitales públicos. Rev calidad Asistencial 2002; 17: 267-72.

10. Martínez DA, Aguayo JL, Soria V, Illán F, Aguirán LM, Pérez-Abad JM, Andreo J. Desarrollo de una vía clínica para el pié diabético. Rev Calidad Asistencial 2003; 18: 235-243.

11. Faglia E, Favales F, Morabito A. New ulceration, new major amputation, and survival rates in diabetic subjects hospitalized for foot ulceration from 1990 to 1993. Diabetes Care 2001; 24: 78-83.

12. Martínez DA, Aguayo JL, Flores B, Morales G, Pérez JM, Alarte JM. Resultados de la hospitalización en pacientes con pie diabético. Cir Esp 2003; 74: 92-96.

13. Monereo S, Pavón I, Vega B, Elviro R, Durán M. Complicaciones de la diabetes mellitus: impacto sobre los costes hospitalarios. Endocrinología 1999; 46: 55-59.

14. Reiber GE, Lipsky BA, Gibbons GW. The burden of diabetic foot ulcers. Am J Surg 1998; 176: S5-S10.

15. Ramsey SD, Newton K, Blough D, Mc Culloch DK, Sandhu N, Reiber GE, Wagner EH. Incidence, outcomes and cost of foot ulcers in patients with diabetes. Diabetes Care 1999; 22: 382-7.

16. Apelqvist J, Tennvall GR, Persson U, Larsson J. Diabetic foot ulcers in a multidisciplinary setting: an economic analysis of primary healing and healing with amputation. J Int Med 1994; 235: 463-71.

17. Real J, Ascaso J. Repercusiones sociales, económicas y sanitarias. En: Camp Faulí A, (Ed): Cuidados del pie diabético. Smith \& Nephew, 2002: 5-10.

18. Crane M, Werber B. Critical pathway approach to diabetic pedal infections in a multidisciplinary setting. J Foot Ankle Surg 1999; 38-1: 30-33. 